SLAC-PUB-11795, hep-ph/yymmnnn

April 2006

\title{
A Universe Without Weak Interactions
}

\author{
Roni Harnik ${ }^{1}$, Graham D. Kribs², and Gilad Perez ${ }^{3}$ \\ ${ }^{1}$ Stanford Linear Accelerator Center, Stanford University, Stanford, CA 94309 \\ ${ }^{2}$ Department of Physics and Institute of Theoretical Science \\ University of Oregon, Eugene, OR 97403 \\ ${ }^{3}$ Theoretical Physics Group, Ernest Orlando Lawrence Berkeley National Laboratory, \\ University of California, Berkeley, CA 94720
}

roni@slac.stanford.edu, kribs@uoregon.edu, gperez@lbl.gov

\begin{abstract}
A universe without weak interactions is constructed that undergoes big-bang nucleosynthesis, matter domination, structure formation, and star formation. The stars in this universe are able to burn for billions of years, synthesize elements up to iron, and undergo supernova explosions, dispersing heavy elements into the interstellar medium. These definitive claims are supported by a detailed analysis where this hypothetical "Weakless Universe" is matched to our Universe by simultaneously adjusting Standard Model and cosmological parameters. For instance, chemistry and nuclear physics are essentially unchanged. The apparent habitability of the Weakless Universe suggests that the anthropic principle does not determine the scale of electroweak breaking, or even require that it be smaller than the Planck scale, so long as technically natural parameters may be suitably adjusted. Whether the multi-parameter adjustment is realized or probable is dependent on the ultraviolet completion, such as the string landscape. Considering a similar analysis for the cosmological constant, however, we argue that no adjustments of other parameters are able to allow the cosmological constant to raise up even remotely close to the Planck scale while obtaining macroscopic structure. The fine-tuning problems associated with the electroweak breaking scale and the cosmological constant therefore appear to be qualitatively different from the perspective of obtaining a habitable universe.
\end{abstract}




\section{Introduction}

Naturalness suggests that the weak interaction ought to be far weaker than observed in our Universe. In this paper we calculate the gross features of a universe without weak interactions. Specifically, we show that by adjusting Standard Model and cosmological parameters we are able obtain a universe that is remarkably similar to our own. This "Weakless Universe" ${ }^{1}$ has big-bang nucleosynthesis, structure formation, star formation, stellar burning with a wide range of timescales, stellar nucleosynthesis up to iron and slightly beyond, and mechanisms to disperse heavy elements through type Ia supernovae and stellar mergers.

Examining the consequences of parameter adjustments on our Universe has a long history (see Refs. [1, 2] for some early discussion). The typical argument is as follows: hold all parameters fixed except for one, vary this one, and find how much it can be changed before the universe with the parameter adjustment appears qualitatively different than without the adjustment. Often "disasters" occur, where the modified universe looks radically different and apparently unsuitable for observers. This suggests that there is an anthropic rationale for parameters to take on values that are consistent with observations in our Universe.

This logic was applied to the cosmological constant (CC) by Weinberg [3]. The CC is by far the most troubling parameter for effective field theorists since (1) it is the most technically unnatural parameter, i.e., it must be tuned to one part in $10^{120}$, and (2) we have no understanding why it should be numerically small but non-zero. As the CC is increased, holding everything else fixed, the universe eventually becomes CC dominated early enough that density perturbations in the universe do not have enough time to grow and go non-linear to form large scale structure. Applying a sensible probability distribution to the value of the CC, Weinberg argued that the CC should be near to the bound set by the anthropic rationale. Remarkably, astrophysical observations are now consistent with a CC that is within about two orders of magnitude of Weinberg's anthropic prediction. This may be viewed as support for varying just the $\mathrm{CC}$ in an ensemble of universes.

Interestingly, even if other astrophysical parameters are varied, such as the amplitude of density perturbations or the baryon asymmetry, the bound on the CC is not significantly relaxed beyond the original Weinberg bound $[4,6]$ (but see also [5]). This analysis assumes these parameters can be freely varied, but the prior probability distribution of these parameters may be sensitive to the details of inflation $[7,8,9]$. Nonetheless, the analysis of [6] may serve as moral justification for varying several low energy parameters simultaneously.

The other technically unnatural parameter, the electroweak scale, also appears to be anthropically limited to a narrow range $[10,11,12,13]$ when only the electroweak scale is varied. We do not disagree with this conclusion. Our viewpoint, however, is orthogonal to these papers. We wish to ask the following question: Is there any universe without electroweak interactions that looks anything like our Universe? Here "any universe" refers to allow ourselves to arbitrarily adjust Standard Model and cosmological parameters. This amounts to adjusting technically natural parameters (since all of the other Standard Model parameters are technically natural, and it is easy to imagine specific models or mechanisms in which cosmological parameters are also determined by technically

\footnotetext{
${ }^{1}$ Throughout this paper we refer to the hypothetical universe without weak interactions as the "Weakless Universe", and the universe that the reader and the authors inhabit as "our Universe".
} 
natural parameters). We should emphasize that there is nothing "wrong" with simultaneously adjusting technically natural parameters of an effective field theory! It is only when the Standard Model is embedded into a precise "ultraviolet completion" when such adjustments may or may not be warranted.

What we will show is that there is no need to anthropically rationalize the small size of the electroweak breaking scale so long as Standard Model and cosmological parameters can be adjusted. This is not a trivial argument, since we require "matching" the Weakless Universe to our Universe including, for instance, stellar fusion with long-lifetime stars. This requirement appears immediately problematic since the reaction $p p \rightarrow D+e^{+}+\nu_{e}$ is absent. We show how this and other apparent problems can be overcome through a careful analysis following suitable parameter adjustments. In the end, the readers must judge for themselves whether the outcome of our exercise provides a sufficiently interesting universe.

We do not engage in discussion of the likelihood of doing simultaneous tunings of parameters nor the outcome of statistical ensembles of parameters. These questions are left up to the ultraviolet completion, such as the string landscape, which is outside of the scope of effective field theory. Instead, we are interested in "running the universe forward" from a time after inflation and baryogenesis through billions of years of evolution. We will exploit the knowledge of our Universe as far as possible, adjusting Standard Model and cosmological parameters so that the relevant microand macro-physical outcomes match as closely as possible. We emphasize that this is really a practical matter, not one of principle, since any significant relaxation of the "follow our Universe" program would be faced with horrendously complicated calculations. Put another way, there is probably a wide range of habitable universes with parameters and structures that look nothing like our Universe. For us, it is enough to find one habitable Weakless Universe about which we can make the most concrete statements, hence matching to our Universe as closely as possible.

We define a habitable universe as one having big-bang nucleosynthesis, large-scale structure, star formation, stellar burning through fusion for long lifetimes (billions of years) and plausible means to generate and disperse heavy elements into the interstellar medium. As a consequence, we will demand ordinary chemistry and basic nuclear physics be largely unchanged from our Universe so that matching to our Universe is as straightforward as possible. We are not aware of insurmountable obstacles extending our analysis to planet formation, habitable planets, and the generation of carbon-based life. Nevertheless, these questions are beyond the scope of this paper, and we do not consider them further.

Finally, we should emphasize from the outset that this paper represents a purely theoretical exercise. There are no (even in principle) experimental predictions. The purpose of our paper is to provide a specific, concrete counter-example to anthropic selection of a small electroweak breaking scale.

\section{The Weakless Universe}

The Weakless Universe is described by a set of parameters that we will relate to the SM and cosmological parameters of our observed Universe. In subsequent sections, we will explain these 
parameter choices in detail, and describe the consequences.

The Weakless Universe contains the gauge group $S U(3)_{c} \times U(1)_{\mathrm{em}}$. This is equivalent to introducing $S U(2)_{L} \times U(1)_{Y}$ with a Higgs mechanism in which the Higgs (mass) ${ }^{2} \rightarrow M_{\mathrm{Pl}}^{2}$. In this sense, the Weakless Universe allows the Higgs (mass) ${ }^{2}$ to take on its most natural value. The strengths of color, electromagnetic, and gravitational forces are fixed by setting

$$
\begin{aligned}
\tilde{\alpha}_{\mathrm{em}}\left(Q^{2}=0\right) & =\alpha_{\mathrm{em}}\left(Q^{2}=0\right) \\
\tilde{\Lambda}_{\mathrm{QCD}} & =\Lambda_{\mathrm{QCD}} \\
\tilde{M}_{\mathrm{Pl}} & =M_{\mathrm{Pl}}
\end{aligned}
$$

where tildes correspond to the parameters of the Weakless Universe. We choose to match the scale of confinement, $\Lambda_{\mathrm{QCD}}$, but this is of course equivalent to matching the strength of the strong force in a perturbative regime $Q^{2} \gg \Lambda_{\mathrm{QCD}}^{2}$. As we will see below, the number of fermion species in the Weakless Universe is different than in our Universe, and thus both the electromagnetic and strong couplings have mild differences in the logarithmic running of their gauge couplings toward the UV.

Given just the color and electromagnetic forces in the low energy effective theory of the Weakless Universe, fermions are vector-like. Thus, there are no constraints on the fermion content at low energies due to anomaly-cancellation. For instance, there is no need for fermions to come in complete generations. Moreover, fermion masses are gauge-invariant without a Higgs mechanism and can be set to whatever values we like. The Weakless Universe contains the up, down, and strange quarks as well as the electron with masses

$$
\tilde{m}_{f}=m_{f} \quad f=u, d, s, e .
$$

The other fermions in our Universe are not present (the reasoning behind keeping the strange quark will be discussed in much more detail in Sec. 3). Of course all of the fermion masses given here are equivalent to having $S U(2)_{L} \times U(1)_{\mathrm{em}}$ broken by a Higgs mechanism with $\tilde{v} \rightarrow M_{\mathrm{Pl}}$ and a large (technically natural) hierarchy between Yukawa couplings of the light species $\lambda_{u, d, s, e} \sim 10^{-22 \rightarrow-19}$ and those of the heavy species $\lambda_{c, b, t, \mu, \tau} \sim 1 .^{2}$ The coincidence that fermion masses and $\tilde{\Lambda}_{\mathrm{QCD}}$ are within a few orders of magnitude in mass in the Weakless Universe is the rephrasing of the coincidence that the electroweak scale is within three orders of magnitude of $\Lambda_{\mathrm{QCD}}$ in our Universe.

The unbroken global symmetries of the Weakless Universe consist of $U(1)_{u} \times U(1)_{d} \times U(1)_{s} \times$ $U(1)_{e}$, generalized from baryon and lepton number of our Universe. ${ }^{3}$ Hence, individual quark number and electron number, not just baryon and lepton number, are conserved. This has significant implications for both the origin of the initial abundances of fermions as well as the stability of bound states of quarks. Quark number is ultimately confined into baryons, as we explain in Sec. 4. The fermion number densities themselves are determined by "fermiogenesis" (generalized from baryogenesis) as will be explained in Sec. 5 .

There are several cosmological parameters that are set in the Weakless Universe as follows. First, the total energy density is given by

$$
\tilde{\Omega}_{\text {total }}=\Omega_{\text {total }}=1,
$$

\footnotetext{
${ }^{2}$ Neutrinos also become heavy in this limit given dimension- 5 operators $(L H)^{2} / M_{\mathrm{Pl}}$ with order one coefficients.

${ }^{3}$ Higher dimensional operators suppressed by the Planck scale violate these individual fermion numbers in the Weakless Universe just like baryon and lepton number in our Universe.
} 
which assumes that the Weakless Universe is flat. We take the flatness, as well as scale-invariant density perturbations with an amplitude $\delta \rho / \rho \sim 10^{-5}$, to arise from inflation, just like our Universe. The total matter density

$$
\tilde{\Omega}_{\text {matter }} \simeq \Omega_{\text {matter }} \simeq 0.23
$$

The difference $\Omega_{\text {total }}-\Omega_{\text {matter }}$ is taken to be vacuum energy, the same as our Universe.

The matter density of the Weakless Universe is fixed to be the same as the matter density of our Universe, so that the transition from radiation domination to matter domination occurs at the same epoch in both Universes. Matter consists of visible baryons (protons and neutrons) and dark matter, analogous to our Universe. Dark matter could consist of free neutral hyperons or a non-baryonic candidate (or both) as we discuss in Sec. 9.

At BBN the visible matter can be described by two parameters, the ratio of the visible baryon abundance to photons $\tilde{\eta}_{b}$ and the ratio of protons to neutron abundance. We take

$$
\tilde{\eta}_{b} \simeq 4 \times 10^{-12} \simeq 10^{-2} \eta_{b}
$$

where we emphasize that this corresponds to just the baryon asymmetry in protons and neutrons, not hyperons. This is taken to be about two orders of magnitude smaller than in our Universe. This judicious parameter adjustment allows the Weakless Universe to have a hydrogen-to-helium ratio the same as our Universe without strong sensitivity to the ratio of the proton to neutron abundance. Hence, the first galaxies and stars are formed of roughly the same material as in our Universe. Moreover, the lower helium abundance that results from the lower baryon asymmetry occurs simultaneous with a substantially increased abundance of deuterium. The much increased deuterium abundance allows stars in the Weakless Universe to ignite through proton-deuterium fusion, explained in detail in Sec. 12.

Given a smaller ratio of visible matter to dark matter, some aspects of large scale structure are affected including the maximum visible mass of galaxies as well as the formation of disks. We will briefly mention these effects in Sec. 8, citing previous results that indicate no disasters occur if the visible baryon abundance in the Weakless Universe is two orders of magnitude smaller than in our Universe.

\section{QCD}

In the Weakless Universe, QCD gets strong and confines at $\tilde{\Lambda}_{\mathrm{QCD}}=\Lambda_{\mathrm{QCD}}$, the same as in our Universe. The number of light(er) quarks is the same as our Universe $(u, d, s)$ so we expect the low energy properties of QCD to be unchanged. In particular, several well-known sum rules in QCD relate these fundamental parameters to the parameters of the chiral Lagrangian, such as the Gell-Mann-Oakes-Renner relation

$$
m_{\pi}^{2} f_{\pi}^{2}=\left(m_{u}+m_{d}\right) \Lambda_{\mathrm{QCD}}^{3}
$$

which implies the product $\tilde{m}_{\pi}^{2} \tilde{f}_{\pi}^{2}=m_{\pi}^{2} f_{\pi}^{2}$ is the same across both universes. This is not surprising since we can construct the low-energy effective Lagrangian involving pions and photons that has 
the same physics in the Weakless Universe as it does in our Universe. Differences in pion masses or couplings between the Weakless Universe and our Universe arise only due to radiative corrections associated with heavier particles and the weak interactions, but these effects are extremely small.

Baryon masses, however, are more subtle. The difference between the proton and neutron mass is determined by the difference in quark masses as well as the electromagnetic splitting. The mass difference in the Weakless Universe is therefore the same as our Universe. The absolute value of nucleon masses, however, receive a large contribution to their mass from gluons as well as an important smaller contribution from strange quarks. The mass fraction attributed to the strange content can be estimated using the approximate $\mathrm{SU}(3)$ flavor symmetry among the light quarks applied to hyperon masses. For example, Ref. [14] find that

$$
\frac{\left\langle N\left|m_{s} \bar{s} s\right| N\right\rangle}{2 m_{N}} \sim 130 \rightarrow 260 \mathrm{MeV}
$$

where the uncertainty depends on whether or not higher order chiral corrections are included. This implies that between about $15 \%$ to $30 \%$ of nucleon mass arises as a result of the strangeness content of the nucleon. While the size of this result is not without controversy (see for example [15]), it is clear that the strange quark does lead to a contribution to the nucleon mass that amounts to between a few to ten or more percent of its mass. This is sufficient to have very significant effects on basic nuclear processes. For instance, deuterium is bound by only $2 \mathrm{MeV}$ which is far smaller than these estimates of the strange contribution to the nucleon mass.

This is the origin of why we hold the strange quark mass fixed in the Weakless Universe. By retaining strange in the low energy effective theory, nucleon masses in the Weakless Universe are the same as in our Universe to very high accuracy (up to effects associated with the charm or heavier quark content of the hadrons which is expected to be an extremely small effect). Sensitive nuclear properties including deuterium binding, the triple- $\alpha$ process, etc., are unaffected. Moreover, given the same baryon masses, pion masses, and couplings, all nuclear physics associated with the residual strong force between nucleons in the Weakless Universe is the same as our observed Universe to extremely high accuracy.

There is, of course, one important difference in the Weakless Universe: hadrons are stable against weak decay, and so free neutrons as well as pions and several strange hadrons are stable. Unraveling the effects of having several stable hadrons in addition to the proton will occupy the next few sections.

\section{Freeze Out Abundances of Hadrons}

Light mesons, which carry an individual fermion (quark) number, such as kaons and charged pions, are stable in the Weakless Universe. Those without quark number, such as the $\pi^{0}$ and $\eta$, are unstable and can decay to photons through the axial anomaly, as in our Universe. Given that there are several stable hadrons in the Weakless Universe, it is important to understand the distribution of the matter abundance in stable hadrons after the QCD phase transition but before BBN.

The result, as we explain below, is that even though the light mesons and the heavier strange baryons (such as the excited states with higher isospin) cannot decay, at finite temperature their 
densities are highly suppressed. Thus, to a very good approximation, the hadronic content of the Weakless Universe just before BBN consists of only protons, neutrons and the lightest stable strange hadron, the $\Lambda_{s}^{0}$ hyperon. Using the chiral Lagrangian it straightforward to see that light mesons and baryons are in thermal equilibrium down to temperatures of order BBN. Thermal equilibrium is maintained through rapid $t$ - or $u$-channel strong interaction processes involving the exchange of light hadrons.

First we show how the universe is driven to a configuration with no light charged mesons. Suppose that at temperatures well below the QCD phase transition a sizable fraction of the up density consisted of $\pi^{+}$(as well as protons and neutrons). Available processes include the interaction of $\pi^{+}$with a neutron that convert into a proton and a $\pi^{0}$ through neutron exchange. Clearly individual quark number is conserved by this process. Then since the neutral pion can quickly decay (at that epoch the corresponding decay rate is much faster than the expansion rate of the Universe), the number density of neutral pions stays at roughly the thermal density, $n_{\pi^{0}} \sim \exp \left(-m_{\pi} / T\right)$. But the thermal density of neutral pions is tiny, so that the inverse process is exponentially suppressed, and so effectively all the charged pions transfer their quark number to baryons in the thermal bath. Similar considerations apply to the charged and neutral kaons once the neutron is replaced with $\Lambda_{s}^{0}$ and $\pi^{0}$ with an $\eta$ meson.

Now suppose that a sizable density of $\Sigma^{+}$baryons is present in the plasma. Processes which involve $\pi^{+} t$-channel exchange will induce $\Sigma^{+}+n \rightarrow \Lambda_{s}^{0}+p$ transitions. The mass difference, $\Delta m$, between the outgoing and incoming particles is much larger than the temperature, $T_{\mathrm{BBN}}$, just before BBN starts

$$
\frac{\Delta m}{T_{\mathrm{BBN}}} \simeq \frac{m_{\Sigma^{+}}-m_{\Lambda_{s}^{0}}}{T_{\mathrm{BBN}}} \sim 80
$$

Similar considerations also apply for the $\Sigma^{0,-}$. Thus at $T_{\mathrm{BBN}}$ the net excess of strange density is equal to the density of $\Lambda_{s}^{0}$ to a very good approximation. To summarize, apart from a sizable fraction of $\Lambda_{s}^{0}$ baryons proportional to the number density of strange quarks, the abundance of stable free mesons and other heavy stable baryons is highly suppressed.

Given that virtually all of the strange quarks are confined into the $\Lambda_{s}^{0}$ hyperon, we now estimate hyperon binding with protons and neutrons. Protons and neutrons bind together only into deuterium, and with a rather small binding energy, $2.2 \mathrm{MeV}$. The binding energy arises through charged and neutral single pion exchange as well as four-fermion operators suppressed by the cutoff scale of the chiral Lagrangian. The $\Lambda_{s}^{0}$, however, cannot exchange of a single pion with a proton or neutron since $\Lambda_{s}^{0}$ is an isospin-singlet. Exchange a single $I=1 / 2$ kaon is possible, but this is highly suppressed by the Yukawa potential of exchanging such a massive scalar particle across the characteristic inverse distance associated with nuclear binding, of order a few to tens of $\mathrm{MeV}$. Another process is the exchange of two pions in a one-loop diagram, but the loop must also contain an isospin-triplet baryon, namely one of the $\Sigma^{ \pm, 0}$ hyperons that are $80 \mathrm{MeV}$ heavier than the $\Lambda_{s}^{0}$. These considerations suggest that the $\Lambda_{s}^{0}$ has far weaker strong interactions with other baryons.

This particular issue has been studied in some detail in our Universe in the context of the search for and study of "hyper-nuclei", i.e., nuclei with one or more hyperons bound to the nucleus [16]. Since weak interactions lead to a relatively slow decay rate, bound states with hyperons can be produced and studied experimentally. Also, theoretical work has been applied to hyper-nuclei 
binding, using various potential models as well as lattice QCD to estimate binding energies. The result of these studies is that "hyper-deuterium" (deuterium with the neutron substituted for a $\Lambda_{s}^{0}$ ) has neither been observed experimentally nor is it expected to be a bound state, consistent with our qualitative analysis above. The lightest experimentally observed bound state with a hyperon is "hyper-tritium", namely a bound state with one proton, one neutron, and one $\Lambda_{s}^{0}$. This is extremely weakly bound with a binding energy of only $130 \mathrm{keV}$ more than that of deuterium. Contrast this with ordinary tritium, which is bound by about $6 \mathrm{MeV}$ more than deuterium. The absence of a bound state of hyper-deuterium combined with the rather weak binding of hyper-tritium suggests that the $\Lambda_{s}^{0}$ plays essentially no role in affecting BBN predictions of the very lightest elements so long as the strange quark number density is not orders of magnitude larger than the up and down quark number density. The $\Lambda_{s}^{0}$ hyperons are therefore expected to become a form of neutral, stable, baryonic dark matter in the Weakless Universe.

\section{$5 \quad$ Fermiogenesis}

The Weakless Universe is assumed to have vanishing total electric charge,

$$
\tilde{Q}_{\text {Universe }}=Q_{\text {Universe }} \simeq 0,
$$

which relates the number density of quarks with electrons,

$$
2 n_{u}-n_{d}-n_{s}=3 n_{e} .
$$

The visible baryon asymmetry at BBN can be related to a different function of the quark number densities

$$
\tilde{\eta}_{b}=\frac{1}{3} \frac{n_{u}+n_{d}-2 n_{s}}{n_{\gamma}}
$$

where the $-2 n_{s}$ accounts for removing hyperons. Given Eqs. (12) and (13), two parameters remain which we can take to be the strange number density $n_{s}$ and the ratio

$$
\left.\frac{n_{n}}{n_{p}}\right|_{\mathrm{eff}}=\frac{n_{u}+2 n_{d}-3 n_{s}}{2 n_{u}+n_{d}-3 n_{s}} .
$$

We showed in Sec. 4 that quark number is ultimately confined into baryons, thus quark number densities can indeed be translated into baryon number densities. For number densities of up, down, and strange that are roughly comparable, we expect $n_{\Lambda_{s}^{0}}=n_{s}$, and the ratio of the remaining up and down quark number densities becomes the ratio of protons to neutrons. These estimates certainly change if there are huge disparities between one quark species to another. For example, if $n_{s} \gg n_{u}, n_{d}$ then the huge excess of strange quarks would have no choice but to bind together into $\Omega^{-}$hyperons. Similarly, if $n_{u}=n_{d}=n_{s}$ equal to high accuracy, then $n_{e} \simeq 0$ by Eq. (12) and the Universe would be composed of just $\Lambda_{s}^{0}$ hyperons. Clearly the relative number densities of quarks plays an essential role in determining the properties of a Weakless Universe.

Like baryon or lepton number in our Universe, these individual quark numbers are free parameters. Unlike our Universe, however, individual quark flavor and lepton flavor is conserved. This 
means that it is not sufficient to simply have a mechanism of baryogenesis, since this relies on weak interactions to distribute the baryon number to the light quark species. Moreover, leptogenesis is also inoperative in the Weakless Universe, since electroweak sphalerons converting lepton number into baryon number are absent.

These considerations imply that baryogenesis must be generalized to fermiogenesis: the origin of the fermion numbers of the Weakless Universe. Of course we do not know the mechanism of baryogenesis in our Universe any more than we know what to match it to in the Weakless Universe. The simplest proposal is to just assume particular initial conditions for the quark numbers (specifically, $n_{s}$ and the ratio $n_{u} / n_{d}$ ), which is tantamount to assuming an excess $B-L$ number in our Universe as the origin of baryons.

Alternatively, mechanisms that do not rely on sphalerons could be employed, such as spontaneous baryogenesis [17] or gravitational baryogenesis [18]. These mechanisms require a source of baryon number violation in our Universe, and hence fermion number violation in the Weakless Universe. It is straightforward to construct models with higher dimensional operators that can populate the fermion numbers as we see fit (with no new technically unnatural parameters) since the flavor structure of these operators is arbitrary. We will not speculate about this in detail, but suffice to say there is nothing particularly unusual about assuming $n_{u} \sim n_{d} \sim \mathcal{O}($ few $) \times n_{s}$ given a relatively flavor democratic fermiogenesis mechanism. In this case, the strange number goes into $\Lambda_{s}^{0}$ and the remaining roughly equal up and down numbers go into roughly equal numbers of protons and neutrons. We will show that this setup (as well as order one variations in the quark number densities) leads to a perfectly habitable Weakless Universe.

\section{$6 \quad \mathrm{BBN}$}

Big-bang nucleosynthesis amounts to a complicated interplay between nucleons binding together through strong interactions in a bath of a huge number photons capable of breaking them up. Given $n_{u} \sim n_{d}$, the Weakless Universe enters the BBN phase with approximately equal numbers of protons and neutrons causing several interesting and important differences on the primordial element abundances. If the visible baryon-to-photon ratio $\tilde{\eta}_{b}$ were the same as in our Universe, then it is easy to estimate that nearly all protons and neutrons get absorbed into helium. A helium-dominated Universe is qualitatively different than our Universe, from the formation of disks in galaxies, to star formation, to the nuclear burning cycle. This may well be an interesting Weakless Universe, but it is not one with which we can reliably calculate. Hence, if we wish to follow our Universe as closely as possible, it is clear that this outcome must be changed.

There are two possibilities. One is to change the ratio of protons and neutrons by adjusting

$n_{u} \neq n_{d}$. For instance, setting $n_{u} \simeq 5 n_{d} / 3$, which corresponds to $n_{p} / n_{n}=7$, roughly reproduces the proton-to-helium ratio in our Universe for the same baryon asymmetry. A second possibility is to leave the number densities of up and down comparable, and instead adjust the visible baryon asymmetry (the total number density of quarks) to be about two orders of magnitude lower than in our Universe. The latter is the route we will take, for reasons that will be clear by the end of this section. 
Numerical calculations are needed to make more precise estimates of BBN. We have modified the standard BBN code [19] to compute the elemental abundances in the Weakless Universe. There are four main differences when computing BBN in the Weakless Universe: First, of course, all weak interactions are decoupled and the neutron is stable. Second, the initial conditions entering the BBN phase depend only on the initial number densities of hadrons that themselves are determined by the number densities of quarks. We will illustrate what happens for a set of three possibilities: $n_{p}=n_{n} \gg n_{\Lambda_{s}^{0}} ; n_{p}=9 n_{n} \gg n_{\Lambda_{s}^{0}} ;$ and $n_{p}=n_{n} / 9 \gg n_{\Lambda_{s}^{0}}$ [These three cases equivalent to $n_{u}=(1,19 / 11,11 / 19) n_{d}$ using Eq. (14)]. In all cases we have neglected the strange quark number density since we already showed that at best it could only weakly bind with deuterium to form hyper-tritium. But since tritium itself is a subdominant component (both in our Universe and in the Weakless Universe) hyper-tritium is even more irrelevant due to its far smaller binding energy. Third, we neglect the effects of having stable mesons around, which is an excellent approximation as we explained above. Finally, there are no neutrinos in the Weakless Universe, and so the number of relativistic degrees of freedom during BBN is slightly smaller causing a slightly slower expansion rate; this leads to a very modest effect on the results, but we nevertheless took it into account.

Given these assumptions, the result of our numerical simulations of BBN are shown in Figs. 1,2,3. The visible baryon-to-photon ratio was varied over a wide range for illustration. Exactly what baryon abundance is optimal for a Weakless Universe depends on initial conditions and on the outcome that we seek.

These calculations lead us to one fascinating result: if the visible baryon asymmetry of the Weakless Universe is lowered to roughly $\tilde{\eta}_{b} \simeq 4 \times 10^{-12}$, a substantial fraction of the synthesized nuclei is deuterium, of order $10 \%$ by mass. This conclusion is not particularly sensitive to the initial number densities of quarks in the range $n_{u} / 9<n_{d}<9 n_{u}$, as the figures show. This can be qualitatively understood in that by reducing the baryon asymmetry we are delaying the initiation of net deuterium production, allowing deuterium to be produced at a lower temperature. The rate of deuterium fusion into ${ }^{4} \mathrm{He}$ is reduced due to the Coulomb barrier, leaving a high abundance of unfused deuterium.

As we will discuss in Sec. 12 below, the existence of a substantial fraction of deuterium provides a mechanism to ignite stars through proton-deuterium fusion, avoiding the proton-proton reaction that is absent in the Weakless Universe. This is the reason that we take the baryon asymmetry to be $\tilde{\eta}_{b} \simeq 4 \times 10^{-12}$ rather than adjusting the number densities of up and down quarks. For this baryon asymmetry and $n_{u} \sim n_{d}$, the resulting hydrogen-to-helium mass fraction is equivalent to our Universe, roughly $Y_{H} / Y_{H e} \simeq 3$. Nevertheless, a far larger fraction of hydrogen is deuterium rather than single protons, so that a hydrogen gas cloud has a composition of about $1 / 4$ deuterium and $3 / 4$ protons by mass. Note also that there is generally a substantial free neutron abundance for $\tilde{\eta}_{b} \simeq 4 \times 10^{-12}$. These electrically neutral relics will behave as form of baryonic dark matter but with a small abundance $\Omega_{n} \lesssim 10^{-4}$.

\section{Chemistry}

In the post-BBN phase of the Universe, the main players in our Universe are electromagnetism and gravity. Both of the these forces are unchanged in the Weakless Universe. The elemental 


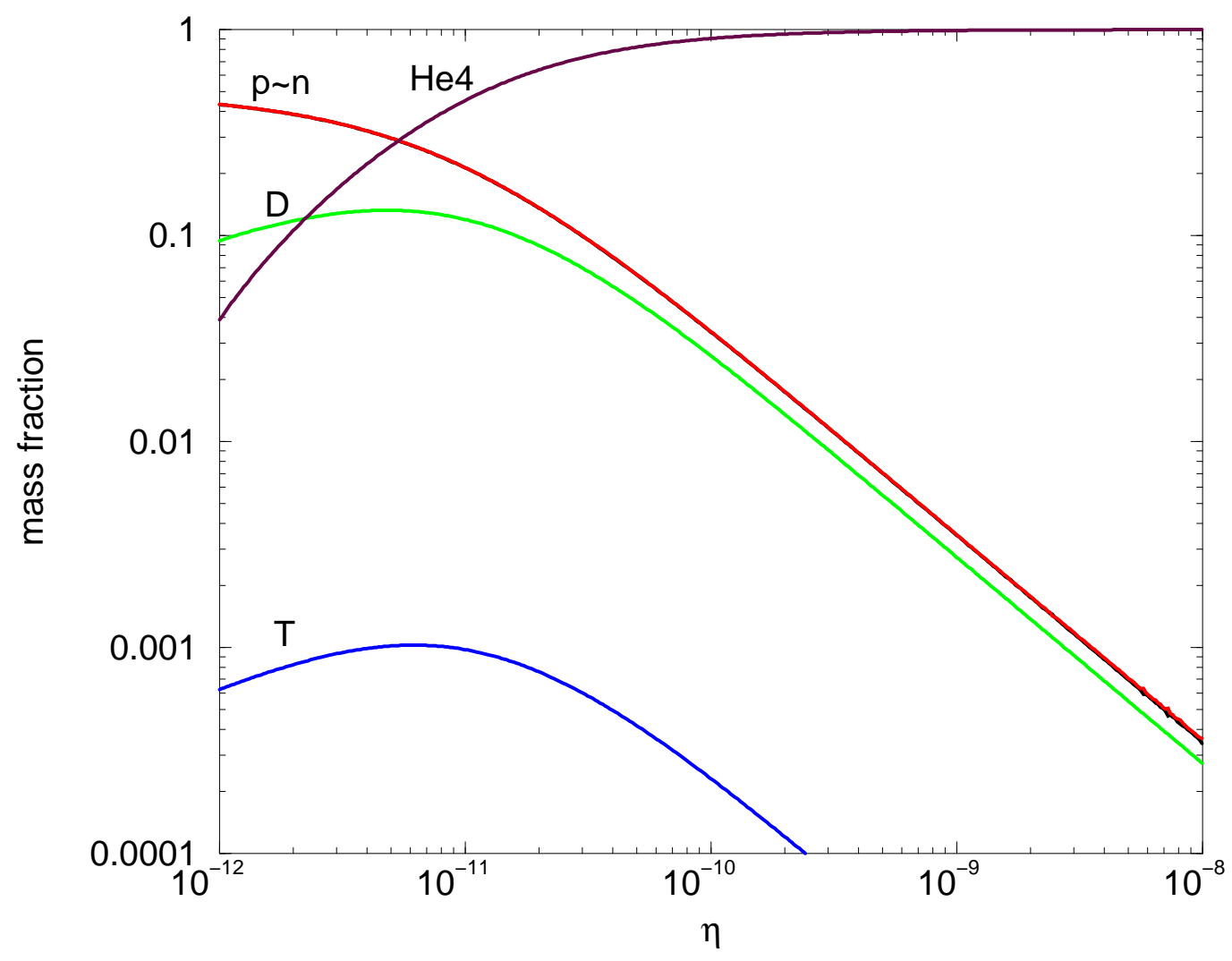

Figure 1: Elemental abundance mass fractions as a function of the visible baryon-to-photon ratio assuming $n_{p}=n_{n} \gg n_{\Lambda_{s}^{0}}$.

abundances of the Weakless Universe have also been matched to our Universe (and are chemically indistinguishable, aside from the irrelevant tiny abundance of lithium). Chemistry in the Weakless Universe is virtually indistinguishable from that of our Universe. The only differences are the higher fraction of deuterium as hydrogen and the absence of atomic parity-violating interactions.

Maintaining this similarity between the Universes relies on having only one stable charged lepton: the electron. The presence of muons or taus (with masses as observed in our Universe) would allow for various exotic chemical properties and nuclear reaction rates. For instance, the Coulomb barrier would be far smaller for atoms with orbiting muons or taus, allowing densepacked molecules and fusion at extremely low temperatures. Though this could be an interesting universe $^{4}$ it does not match our Universe and so we choose to remove muons and taus from the Weakless Universe.

Other more benign effects occur if the heavier quarks $(c, b, t)$ were present in the Weakless Universe. Given that individual quark number is conserved, the lightest baryons carrying a heavy

\footnotetext{
${ }^{4}$ Consider a universe with $\tilde{m}_{\tau}=m_{\tau}$ with baryons dominantly in ${ }^{4} \mathrm{He}$. A ${ }^{4}$ He nucleus with one $\tau^{-}$and one electron will behave chemically like hydrogen. This is because the double-charged nucleus will be screened to single-changed by the tight orbital of the $\tau^{-}$. This provides a potential mechanism to allow ${ }^{4} \mathrm{He}$ fusion at much lower temperatures as well as forming hydrogen-like molecules that are important for star formation, see Sec. 11.
} 


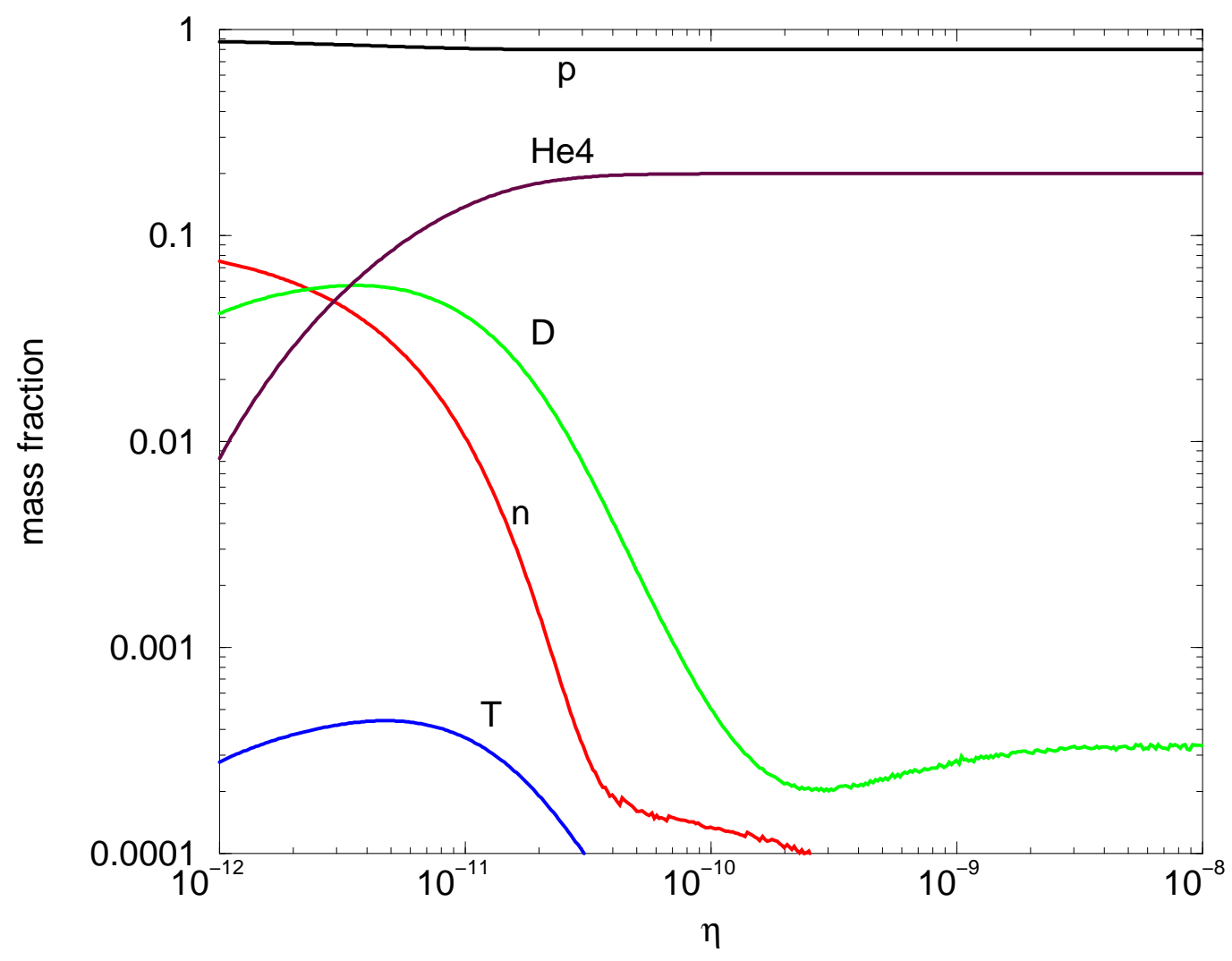

Figure 2: Same as Fig. 1 except $n_{p}=9 n_{n} \gg n_{\Lambda_{s}^{0}}$.

quark are stable. This means in addition to protons, neutrons, and $\Lambda_{s}^{0}$ hyperons, there would be several new stable baryons including $\Lambda_{c}^{+}, \Lambda_{b}^{0}$ and $\Lambda_{t}^{+} \cdot{ }^{5}$ If these exotic stable baryons were in significant abundance in the Weakless Universe, there would be numerous anomalously heavy isotopes of hydrogen (and heavier elements). These are not obviously an impediment to successful $\mathrm{BBN}$ or star formation, but it would change the details of stellar nucleosynthesis reactions in ways that we are not able to easily calculate. Again, following our program of matching to our Universe as closely as possible, we eliminate this problem by insisting that the Weakless Universe is devoid of these heavy quarks.

\section{Matter Domination and the Growth of Density Perturbations}

One extremely important epoch in our Universe is the era of matter domination. This is when density perturbations are able to grow linearly and subsequently collapse to form structure. This must occur before any structure-destroying epoch takes place, such as if a cosmological constant eventually dominates the energy density. Matching to our Universe suggests that we take $\tilde{\Omega}_{\text {total }}=$

\footnotetext{
${ }^{5}$ Several species of isospin non-singlets are also stable, but their primordial abundance is expected to be far smaller than the isospin-singlets for the same reason that the $\Sigma^{ \pm, 0}$ abundance is far smaller than the $\Lambda_{s}^{0}$ as discussed above.
} 


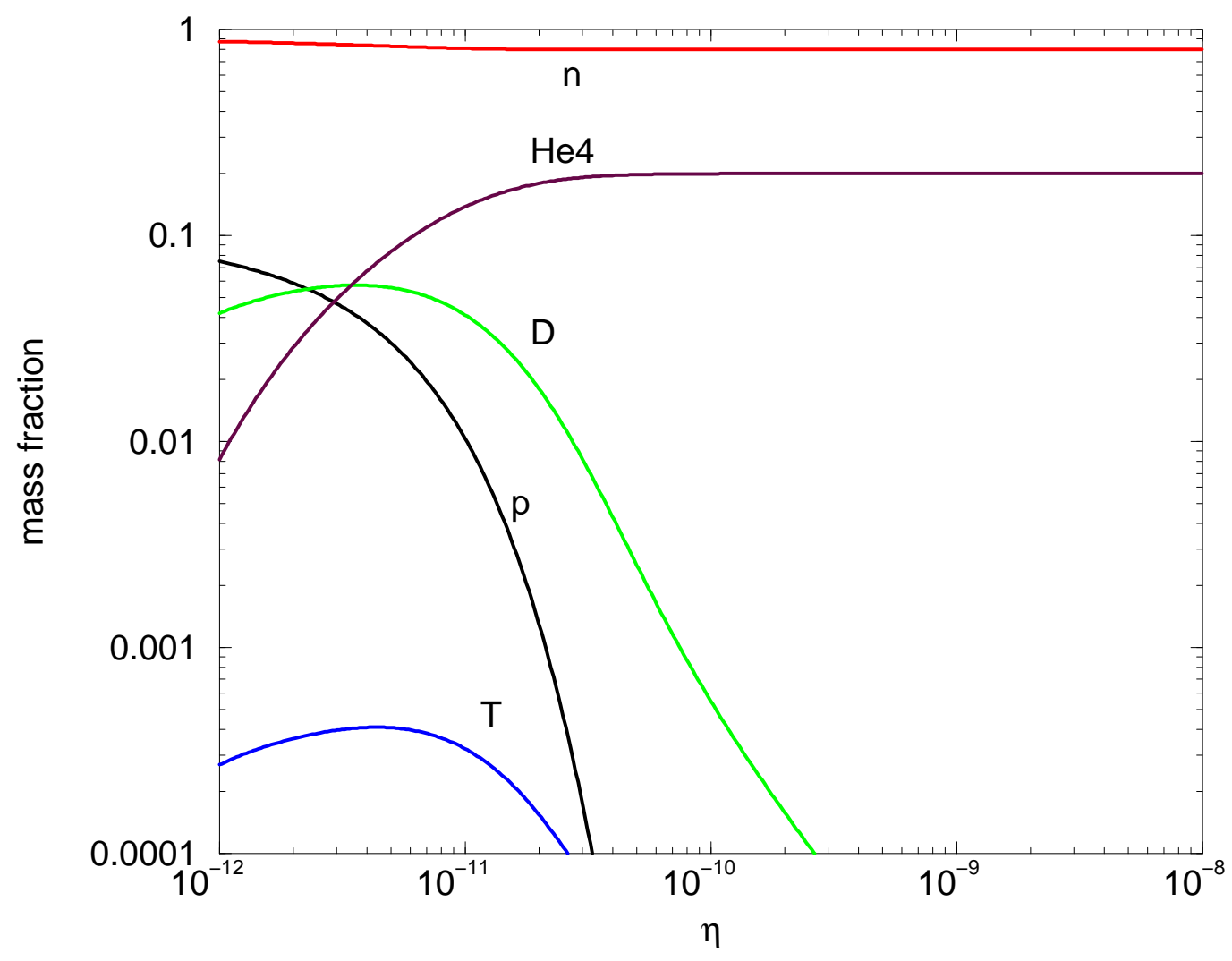

Figure 3: Same as Fig. 1 except $n_{p}=n_{n} / 9 \gg n_{\Lambda_{s}^{0}}$.

$\Omega_{\text {total }}=1$ and $\tilde{\Omega}_{\text {matter }}=\Omega_{\text {matter }} \simeq 0.23$. There is certainly no need for a cosmological constant to dominate the latter epoch of the Weakless Universe; instead it could be an open universe with $\tilde{\Omega}_{\text {total }} \simeq 0.23$, or dark matter could make up the difference between a closed universe and the baryons and radiation. Both of these possibilities, however, are expected to have "higher order" effects on the evolution of our Universe (for instance, a larger dark matter abundance implies matter-domination occurs at a slightly earlier epoch). Matching to our Universe as closely as possible suggests that we take the Weakless Universe to have the cosmological parameters as given originally in Sec. 2.

Since we have chosen to hold the $n_{H} / n_{H e}$ fraction constant with $\tilde{\eta}_{b} \sim 4 \times 10^{-12}$, the visible baryon density in the Weakless Universe is similarly reduced, $\Omega_{\text {visible baryons }} \sim 10^{-4}$. The smaller visible baryon abundance implies we should expect an overall decrease in the number density of stars in galaxies, although calculating precisely the scaling relies on knowing detailed dynamics of galaxy formation.

The maximum size of a given large scale structure to form in the universe depends on the scale of the initial perturbation and the time with which it can grow. Ref. [20] found that the maximum mass $M^{\max }$ that any structure which can form in the universe can be expressed simply as

$$
M^{\max } \lesssim M_{\mathrm{Pl}}^{3} \delta_{0}^{3 / 2} \rho_{\Lambda}^{-1 / 2}
$$


where $\delta_{0}$ is the size of the density perturbation and $\rho_{\Lambda}$ is the size of the cosmological constant density. Putting in values for our Universe, one finds $M^{\max } \lesssim 10^{16} M_{\odot}$. Our galaxy contains about $10^{11} M_{\odot}$ baryons, which implies one can form a lower bound on the ratio of visible matter to dark matter of about $10^{-5}$ so that galaxies like our own are formed. The Weakless Universe with $\tilde{\Omega}_{\text {visible baryons }} / \tilde{\Omega}_{\text {dark matter }} \sim 10^{-3}$ clearly satisfies this bound.

Once structures of galactic size are able to form, the gas of baryons must be able to cool sufficiently into a rotationally supported disk from which stars can be created. Ref. [6] found that there is a more stringent lower bound on the ratio of visible matter to dark matter from this constraint. The precise numerical value of the lower bound depends on the mechanism of cooling (hydrogen line cooling or molecular hydrogen cooling) and the virial temperature of the galaxy or structure. Given these uncertainties, they found the lower bound to be of order $3 \times 10^{-3} \rightarrow$ $10^{-4}$. Ref. [6] also showed that molecular viscosity can relax this lower bound by another order of magnitude, so a fair conclusion from this analysis is that one begins to run into trouble only when the baryon to matter density ratio falls below about $10^{-4}$. Again, the Weakless Universe satisfies this bound.

\section{Dark Matter Candidates}

We have already remarked that free neutrons and free $\Lambda_{s}^{0}$ hyperons would behave as baryonic dark matter. This is amusing, since the abundance of (baryonic) dark matter becomes closely connected with the matter abundance in the Universe. However, given that the total baryon abundance is two orders of magnitude smaller in the Weakless Universe, this dark matter is insufficient to cause the Universe to become matter-dominated at a time the same as (or close to) our Universe. The Weakless Universe therefore needs non-baryonic dark matter to match to our Universe.

The usual candidates for non-baryonic dark matter contemplated for our Universe are not necessarily effective in the Weakless Universe. Weakly interacting massive particles (WIMPs) with an electroweak scale mass whose abundance is determined by the usual thermal freeze-out after electroweak decoupling are obviously unsuitable since electroweak interactions are absent! (This does not preclude a non-thermal source of $100 \mathrm{GeV}$ mass mass particles, but the mass scale here is clearly arbitrary.) It is straightforward to add a dark matter sector that is unconnected with weak interactions, such as axions or primordial black holes. This is the working assumption that we have in mind for the Weakless Universe.

Yet another possibility is to deviate from the parameters of the Weakless Universe in the following way. Take $\tilde{\Omega}_{\text {baryons }}=\tilde{\Omega}_{\text {matter }}$ with $n_{u}=n_{d}=1.0001 n_{s}$. Then the vast majority $(99.99 \%)$ of quarks are confined into $\Lambda_{s}^{0}$ hyperons, which act as baryonic dark matter. The reminder go into "visible" protons and neutrons with an abundance that remains of order $10^{-4}$ so that BBN largely follows Fig. 1. This Universe is harder to calculate, since such a large abundance of $\Lambda_{s}^{0}$ hyperons during BBN may well lead to a more significant abundance of exotic elements including hyper-tritium and hyper-helium. While we expect this would be a perfectly viable alternative, the modifications to BBN and stellar nucleosynthesis make it harder for us to match to our Universe and so we will not consider this amusing possibility any further. 


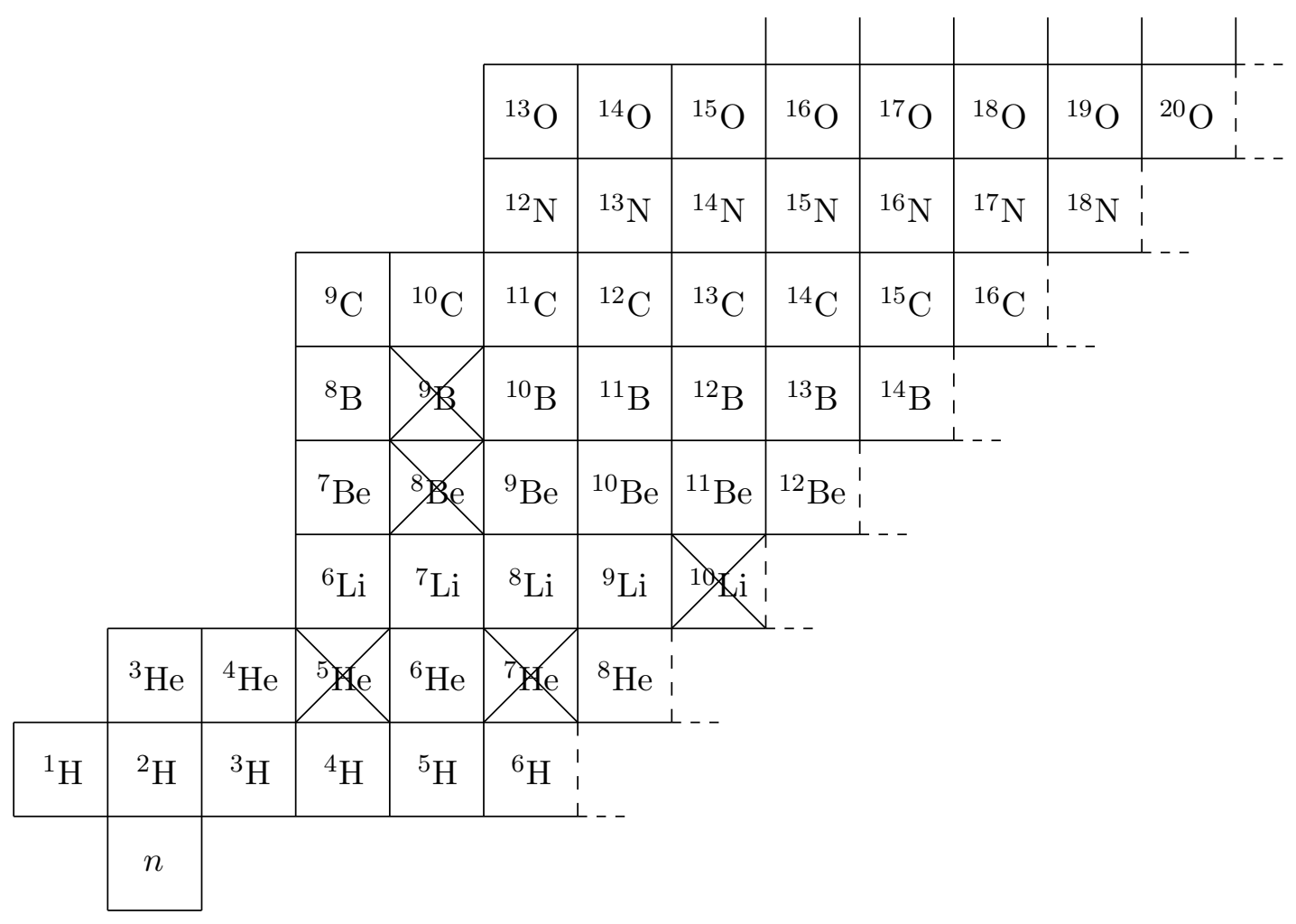

Figure 4: The expected isotopic stability table in the Weakless Universe. Only isotopes with $A \leq 20$ and $Z \leq 8$ are shown. Reliable knowledge of neutron-rich isotopes is not extensive for most low $Z$ elements; those not shown on the right-hand side are not necessarily unstable to neutron emission. The proton-rich isotopes not shown on the left-hand side are unstable to proton emission. The crossed-out elements are unstable to proton, neutron, or $\alpha$ emission.

\section{Heavy Element Isotopic Stability}

In our Universe, only a narrow band in the atomic mass-number $(A-Z)$ plane contain stable elements. In the Weakless Universe, the band is much wider, extending out to the edge of the "valley of stability" well known from nuclear physics. In particular, since both $\beta$-capture and $\beta$ decay are absent, a much wider range of proton-rich and neutron-rich isotopes are stable. Using tables from Ref. [21], we can estimate the isotopic stability table for the Weakless Universe by relabeling all previously unstable (to $\beta$-capture or $\beta$-decay) isotopes as being stable. This is shown in Fig. 4 for elements up to oxygen. Elements which are unstable to weak decay are presumably stable against proton, neutron, or $\alpha$ emission. $^{6}$

The conservation of both proton number and neutron number, combined with the smaller total number of neutrons over protons in a primordial gas cloud implies that proton-rich isotopes are likely to be populated. Recall, the primordial gas cloud is expected to contain $75 \%$ hydrogen and

\footnotetext{
${ }^{6}$ However, we cannot rule out tiny mass splittings that might allow decay via strong interactions that happened to be accidentally much weaker than weak interactions due to kinematic suppression.
} 
$25 \%$ helium, in which about $1 / 4$ of the hydrogen is deuterium by mass. None of these primordial elements has more neutrons than protons. Combine this initial condition with the fact that isotopes with an equal or excess number of protons over neutrons never spontaneously emit a neutron implies that stellar nucleosynthesis is expected to create elements along the $A \leq 2 Z$ side of the valley of stability.

The critical point in which the last element with equal numbers of protons and neutrons $(A=$ $2 Z)$ is stable in the Weakless Universe against decay into a $Z-1$ element and a proton is ${ }^{76} \mathrm{Sr} .{ }^{7}$ This is well above iron, suggesting that stellar fusion is perfectly capable of creating nearly the entire first half of the periodic table. In our Universe, by contrast, the last stable element on the $A=2 Z$ line is ${ }^{40} \mathrm{Ca}$. To produce elements above ${ }^{76} \mathrm{Sr}$ in the Weakless Universe requires stellar nucleosynthesis reactions that emit neutrons. In our Universe, one known candidate reaction is ${ }^{22} \mathrm{Ne}+\alpha \rightarrow{ }^{24} \mathrm{Mg}+n$ (that would occur in the late stage of neon burning) leading to a " $s$-process" to synthesize heavier elements. This requires, however, weak interactions to get to the neutron-rich side of neon $\left(A_{\mathrm{Ne}}>20\right)$. While we cannot rule out the possibility of other reactions that emit neutrons in the later stages of stellar burning, they appear to be unlikely since they would follow a path away from the bottom of the valley of stability. It appears unlikely to produce any significant abundance of elements above about $Z=38(\mathrm{Sr})$.

In our Universe, superheavy elements well above iron are created in supernovae. In a later section we will discuss supernovae in the Weakless Universe, but clearly the conservation of proton and neutron number, combined with the absence of weak decays, prevents the usual pathways to creating superheavy elements. There are amusing consequences of the absence of heavy elements and radioactive decay. For instance, the existence of a molten core that is continually heated by radioactive uranium and thorium in earth-like planets would not occur in the Weakless Universe. Plate tectonics, volcanos, geothermal heat, etc., would not exist billions of years after planets form. Nevertheless, we do not view this difference with our Universe as anything more than a curiosity.

\section{Star Formation}

In order for a dilute gas to collapse into a bound object it must lose energy, satisfying the virial theorem. Heuristically, one imagines that an atom with some initial kinetic energy falls into a potential well. The only way for the atom to be bound into the potential without climbing out is for it to lose energy as it falls inside. The first condition for star formation is thus an efficient cooling mechanism whereby the gas can radiate energy out of the system.

There are several mechanisms for a gas cloud to lose energy. An efficient mechanism to cool a gas cloud down to temperatures of order $T \sim 1 \mathrm{eV}$ is line radiation from primordial hydrogen and helium. This corresponds to a Jeans mass

$$
M_{J} \simeq 10^{6}\left(\frac{T}{1 \mathrm{eV}}\right)^{3 / 2}\left(\frac{n}{10^{4} \mathrm{~cm}^{3}}\right)^{-1 / 2} M_{\odot} .
$$

Clearly, to get anywhere close to stellar mass objects, it is crucial to lower the temperature of the

\footnotetext{
${ }^{7}$ Actually, there is one higher isotope that is stable, ${ }^{84} \mathrm{Mo}$, but it is separated from ${ }^{76} \mathrm{Sr}$ by gaps at $A=78,80,82$ whereas all of the $A=2 Z$ elements at and below ${ }^{76} \mathrm{Sr}$ are stable.
} 
gas even further. The density of the gas, $n$, must also increase, but this cannot continue arbitrarily, since the gas cloud is expected to form a rotationally supported disk.

To lower the gas temperature below what hydrogen or helium line radiation can accomplish requires either other gas components (elements created from the first stars, i.e., a sub-dominant metallicity component with line radiation at lower energies) or another mechanism. The first stars would not have other gas components, and it is generally believed that molecular hydrogen plays an important role to cool the gas to of order $T \sim 0.01 \mathrm{eV}$ through soft vibrational modes. Molecular hydrogen is typically formed through gas-electron collisions where free electrons act as catalysts. In Ref. [22] it was shown that a non-equilibrium abundance of molecular hydrogen is expected to form in the gas clouds of the early Universe that lead to the first stars.

This is the ultimate origin of our requirement that the outcome of BBN lead to a chemical composition in the Weakless Universe that is similar to our Universe. For instance, if baryons were buried dominantly in helium, such as would exist for a baryon-to-photon ratio equal to our Universe (with $n_{u} \simeq n_{d}$ ), no molecular transitions would be available to cool the gas below what helium line radiation could accomplish since helium does not form molecules. Sub-dominant components may well allow for helium to condense. For instance, with $\tilde{\eta}_{b}=\eta_{b} \simeq 5 \times 10^{-10}$ and $n_{u}=n_{d} \gg n_{s}$, we find that BBN results in about $97 \%$ by mass in helium and about $2 \%$ by mass in hydrogen. Hence, there is a sub-dominant component that could allow for cooling below $T \sim \mathrm{eV}$. However, helium itself does not begin to fuse until the temperature of the gas is much higher than for hydrogen fusion, and this ultimately leads to far fewer stars that live only as long as helium burning permits, which is tens of millions of years. Again, this may well lead to an habitable universe, but it is not one with which we can make reliable predictions due to the difficulties of simulating helium gas cloud collapse, ignition, etc.

\section{Stellar Nucleosynthesis}

In our Universe, a collapsing gas cloud with hydrogen begins to fuse by the weak interaction

$$
p+p \quad \rightarrow \quad \mathrm{D}+e^{+}+\nu_{e}
$$

once the temperature of the core rises above about $10^{7} \mathrm{~K}$. This reaction is obviously absent in the Weakless Universe and naively appears to be serious bottleneck to stellar ignition. However, we showed that for $\tilde{\eta}_{b} \simeq 4 \times 10^{-12}$ and $n_{u} \simeq n_{d}$, the synthesized elements of BBN included a large fraction of deuterium.

The presence of a much higher fraction of deuterium allows collapsing clouds of hydrogen gas to skip the slow, weak interaction mediated process (17) and go directly to

$$
p+\mathrm{D} \rightarrow{ }^{3} \mathrm{He}+\gamma
$$

Subsequent burning occurs by the standard chains

$$
\begin{aligned}
{ }^{3} \mathrm{He}+{ }^{3} \mathrm{He} & \rightarrow{ }^{4} \mathrm{He}+2 p \\
\mathrm{D}+{ }^{3} \mathrm{He} & \rightarrow{ }^{4} \mathrm{He}+p
\end{aligned}
$$


to generate energy through fusion up to ${ }^{4} \mathrm{He}$. The hydrogen-to-helium fusion chain in the Weakless Universe is therefore effectively

$$
2 p+2 \mathrm{D} \rightarrow{ }^{4} \mathrm{He}+2 p
$$

where the protons act as catalysts to fuse deuterium into ${ }^{4} \mathrm{He}$. The deuterium burning reaction is very fast and thus can change the dynamics of stellar burning in the Weakless Universe. Below we remark on how this leads to modest changes in the stellar mass-lifetime relation.

In our Universe, burning above helium is "blocked" by the absence of stable elements at atomic mass numbers $A=5$ and $A=8$. In the Weakless Universe, the $A=5$ gap persists, namely ${ }^{5} \mathrm{Li}$ is unstable to proton emission and ${ }^{5} \mathrm{He}$ is unstable to neutron emission (see Fig. 4). The $A=8$ gap partially persists given that ${ }^{8} \mathrm{Be}$ is unstable to breakup into two $\alpha$ 's, but ${ }^{8} \mathrm{~B},{ }^{8} \mathrm{Li}$, and ${ }^{8} \mathrm{He}$ are all stable. Of these, ${ }^{8} \mathrm{Li}$ and ${ }^{8} \mathrm{He}$ are unlikely to play any role. This is because the neutron-rich side of the valley of stability is virtually impossible to reach at this point in stellar nucleosynthesis since it requires starting with neutron-rich ingredients, of which there are none. ${ }^{8}$

Once the stellar core temperature gets high enough, the helium can begin to fuse via the famous triple- $\alpha$ process

$$
3\left({ }^{4} \mathrm{He}\right) \rightarrow{ }^{8} \mathrm{~B}+{ }^{4} \mathrm{He} \rightarrow{ }^{12} \mathrm{C}+\gamma
$$

where a non-equilibrium abundance of ${ }^{8} \mathrm{~B}$ is built up and consumed into ${ }^{12} \mathrm{C}$. Then, carbon can be pushed further up the nucleotide table through reactions such as

$$
{ }^{12} \mathrm{C}+{ }^{4} \mathrm{He} \rightarrow{ }^{16} \mathrm{O}+\gamma
$$

that occur our Universe, as well as new "proton clumping" reactions such as

$$
\begin{aligned}
& { }^{12} \mathrm{C}+p \rightarrow{ }^{13} \mathrm{~N}+\gamma \\
& { }^{13} \mathrm{~N}+p \rightarrow{ }^{14} \mathrm{O}+\gamma .
\end{aligned}
$$

In the Weakless Universe, ${ }^{13} \mathrm{~N}$ and ${ }^{14} \mathrm{O}$ are stable (see Fig. 4) but ${ }^{15} \mathrm{~F}$ is not, and so this particular proton clumping trajectory stops at oxygen.

New reaction chains to fuse up to proton-rich isotopes are possible. For example, in the Weakless Universe there are trajectories that lead to ${ }^{9} \mathrm{C},{ }^{10} \mathrm{C}$, and ${ }^{11} \mathrm{C}$ which are all stable. The starting point to enter the proton-rich side is ${ }^{3} \mathrm{He}$. Naively one might guess the reaction

$$
{ }^{3} \mathrm{He}+\mathrm{D} \rightarrow{ }^{5} \mathrm{Li}+\gamma
$$

but this is far slower than $p+\mathrm{D}$ reaction as well as ${ }^{5} \mathrm{Li}$ being unstable to decay into ${ }^{4} \mathrm{He}+p$. This leaves only

$$
{ }^{3} \mathrm{He}+{ }^{4} \mathrm{He} \rightarrow{ }^{7} \mathrm{Be}+\gamma
$$

\footnotetext{
${ }^{8}$ There is a small but non-trivial mass fraction of tritium, of order $0.1 \%$ [see Fig. 1]. However, the $p+T \rightarrow{ }^{4} \mathrm{He}+\gamma$ reaction is by far the fastest reaction for tritium burning, and thus we expect this neutron-rich isotope to long since have disappeared into ${ }^{4} \mathrm{He}$.
} 
as the starting point into the proton-rich side of the isotopes since ${ }^{7} \mathrm{Be}$ is stable in the Weakless Universe. Proton-clumping interactions such as

$$
\begin{array}{r}
{ }^{7} \mathrm{Be}+p \rightarrow{ }^{8} \mathrm{~B}+\gamma \\
{ }^{8} \mathrm{~B}+p \rightarrow{ }^{9} \mathrm{C}+\gamma
\end{array}
$$

can push up to ${ }^{9} \mathrm{C}$, but no further, since ${ }^{10} \mathrm{~N}$ is not stable. Helium-clumping interactions such as

$$
\begin{array}{r}
{ }^{7} \mathrm{Be}+{ }^{3} \mathrm{He} \rightarrow{ }^{10} \mathrm{C}+\gamma \\
{ }^{7} \mathrm{Be}+{ }^{4} \mathrm{He} \rightarrow{ }^{11} \mathrm{C}+\gamma \\
{ }^{8} \mathrm{~B}+{ }^{3} \mathrm{He} \rightarrow{ }^{11} \mathrm{~N}+\gamma \\
{ }^{8} \mathrm{~B}+{ }^{4} \mathrm{He} \rightarrow{ }^{12} \mathrm{~N}+\gamma
\end{array}
$$

also produce proton-rich isotopes of carbon and nitrogen. Further proton-clumping and heliumclumping can populate proton-rich isotopes of oxygen and higher. The extent to which this series of reactions or the usual triple- $\alpha$ process dominates to generate carbon and other higher $Z$ elements depends on nuclear cross-sections, core temperatures, and detailed modeling of stellar cores. It would be extremely interesting to simulate stellar fusion given the initial abundances of protons, deuterium, and helium in the absence of weak interactions. This is, however, beyond the scope of this paper.

\section{Stellar Lifetimes}

A star is a self-controlled reactor in steady-state equilibrium. Its lifetime, once in steady-state, does not depend directly on the rates of micro-physical processes but rather on its gross thermodynamical properties. This means that even though stars in the Weakless Universe begin fusion by the fast reaction (18), stellar lifetimes can nevertheless be billions of years long.

Steady-state equilibrium implies that the energy that is radiated away from the star's surface must equal the energy produced in its interior. This internal energy production comes from gravitational collapse and nuclear reactions. Starting with a gas cloud of mass $M$ and a certain chemical composition, steady-state equilibrium may be stated as an equality of luminosities

$$
L_{\star}(T, M, \ldots)=L_{\text {nuc }}(T, M, \ldots)+L_{\text {col }}(T, M, \ldots)
$$

where $L_{\star}$ is the stellar luminosity, $L_{\text {nuc }}$ is the rate of nuclear energy production and $L_{\text {col }}$ is that from gravitational collapse.

The luminosity of a star, $L_{\star}$, is set by its large scale properties that are determined by hydrostatic and thermal equilibrium. Hydrostatic equilibrium requires that gravitational pressure must balance against a combination of thermal gas pressure, electron degeneracy pressure, and radiation pressure. In the stars considered here, as is the case in our Sun, the dominant pressure is the gas pressure which can be approximated by the ideal gas law, $P \propto \rho T$. Hydrostatic equilibrium leads to a decreasing temperature gradient from the stellar core to the surface.

The details of the temperature gradient are also determined by the mechanism and rate of energy transport from the core. If the opacity is low enough, the dominant mechanism to transport energy 
from the core to the surface is by radiation, as occurs for example for the Sun. However, during the protostellar phase, when the Sun was much younger and colder, the opacity was much higher. This inability to efficiently transport energy by radiation leads to an instability in the pressure gradient of the star, causing convection to be the dominant energy transport mechanism. Stars whose energy transport mechanism is convection are said to be in the Hayashi phase. Convection is generally a more efficient transport mechanism, and so stars in their Hayashi phase are more luminous than they are in their later radiative phase ${ }^{9}$.

Given a detailed model for the equation of state and energy transport in the star one can calculate the stellar radius and temperature profile (generally using a numerical simulation). The stellar luminosity $L_{\star}$ is given by black-body radiation

$$
L_{\star}=\left(4 \pi R^{2}\right) \sigma T_{\mathrm{eff}}^{4}
$$

where $\sigma$ is the Stefan-Boltzmann constant and $T_{\text {eff }}$ is roughly given by the surface temperature of the star. To gain some appreciation for the behavior of $L_{\star}$ with time, we can follow the evolutionary track of a solar mass star [23] as is described schematically in Fig. 5. A young star in the convective Hayashi phase is initially much more luminous than our Sun, however its luminosity decreases with time. Then the central temperature increases but the surface temperature remains roughly constant. The decreasing radius leads to a drop in luminosity. At some point radiation becomes the dominant energy transport mechanism and the surface temperature begins to rise as the star continues to collapse and the luminosity begins to climb with increasing core temperature. Stars with smaller masses follow a roughly parallel track, but with a smaller luminosity compared to a solar mass star. However, the onset of the radiative phase is delayed for stars with lower masses.

Knowing at what rate a star radiates energy we now analyze the internal energy sources, i.e., the right hand side of Eq. (34). The nuclear reaction rate is set by the details of the nuclear process in question. Basically, it is set by the tunneling probability through a Coulomb barrier at a given temperature. Any such rate will be a steeply climbing function of temperature. At any given temperature, reactions involving the strong force will be much more rapid than those involving the weak force, however this does not mean the star will be short-lived. Instead, this allows the star to burn at a lower temperature.

When the star is young and cold, the nuclear reaction rates are exceedingly small. The only source of energy satisfying Eq. (34) is from gravitational collapse. As the star contracts, half of the gravitational potential energy is converted into heat, the other half radiated away, satisfying the virial theorem. As the star continues to collapse, the central temperature increases, the nuclear reaction rates rapidly increase, supplying a larger fraction of energy to the star. This slows the gravitational collapse, maintaining thermal equilibrium. The gravitational collapse halts once nuclear reactions saturate the stellar luminosity. When this occurs, a star is said to be in a steadystate that defines the main sequence of stellar populations. A star on the main sequence continues to shine without changing its gross features until it consumes its burnable nuclear material. The lifetime of a star in steady state is thus

$$
t_{\star}=\frac{\text { Burnable Energy }}{\text { Luminosity at Steady State }} .
$$

\footnotetext{
${ }^{9}$ We thank Savas Dimopoulos and Jay Wacker for discussions about the Hayashi phase.
} 


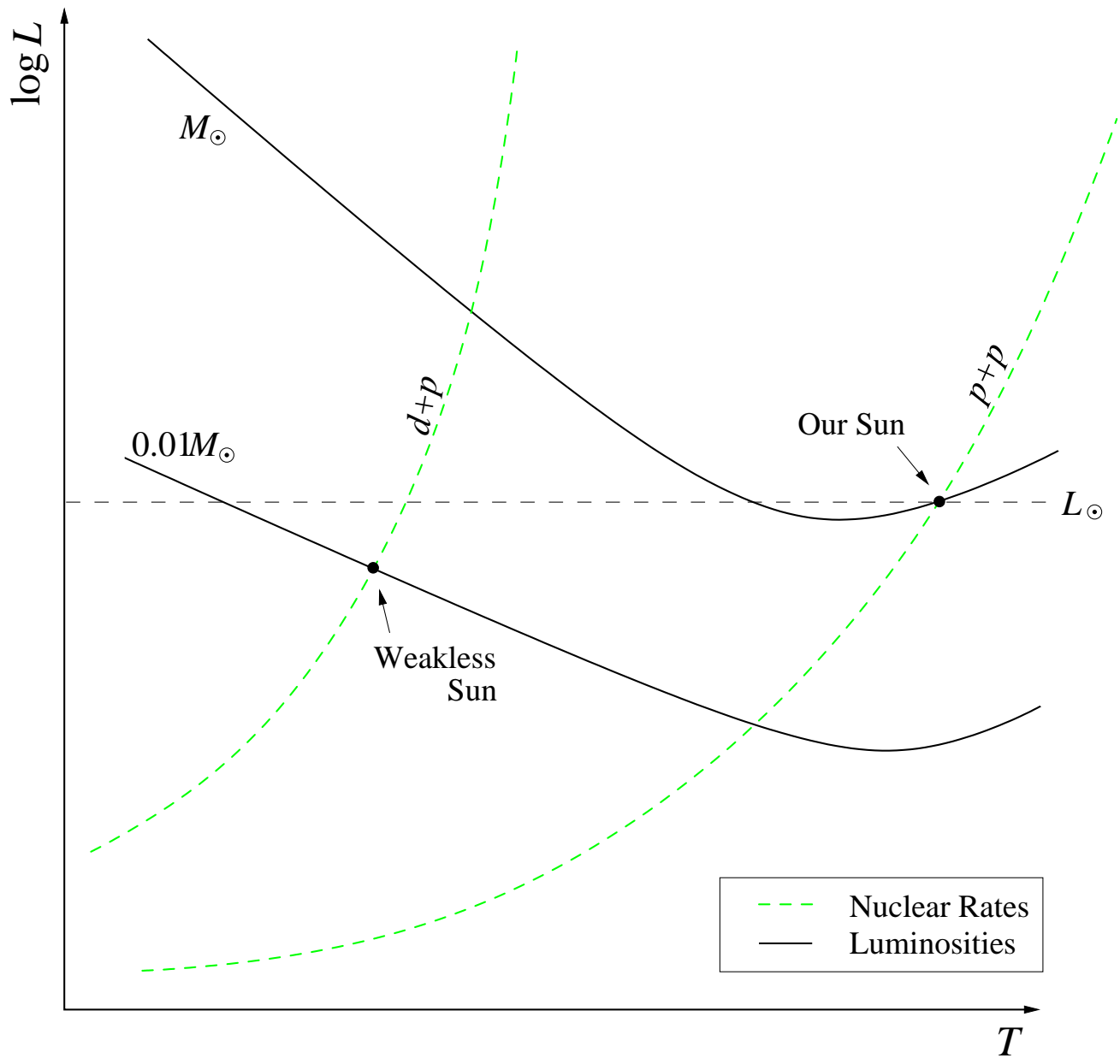

Figure 5: A schematic plot of the stellar luminosity (black) and nuclear burning rate (red) on a log scale as a function to the stellar core temperature. The phase of decreasing luminosity is the Hayashi phase, when convection is dominant. Stars will remain in a steady state when the luminosity and nuclear rate are equal. The lifetime of the star in steady state is the ratio of burnable energy to the steady state luminosity. In the Weakless Universe one can get long lives stars if the primordial deuterium abundance is higher than in our universe, increasing the amount of burnable fuel. The longest living stars, i.e. with low luminosity, are of order $0.01-0.02 M_{\odot}$. 
In the Weakless Universe there are two classes of long-lived stars: deuterium burning and ${ }^{3} \mathrm{He}$ burning.

Consider first stars that burn deuterium. In our Universe, it is believed that brown dwarfs, which are stars in the range $\sim 0.01-0.2 M_{\odot}$, burn deuterium for millions of years. Due to the low primordial deuterium abundance of our Universe these stars never reach a deuterium burning steady-state $[24,25]$. In the Weakless Universe, the deuterium abundance is much higher and thus a deuterium burning star can easily achieve steady-state equilibrium. Detailed simulations of deuterium burning in brown dwarfs has been done for our Universe [24]. This simulation was repeated by Burrows [26] for the Weakless Universe with a primordial deuterium abundance of $y_{d} \sim 0.1 .^{10}$ For example, a star with a mass of $0.02 M_{\odot}$ burned deuterium in steady-state for roughly 7 billion years with a luminosity of $0.025 L_{\odot}$. Less massive stars burned for a longer time. ${ }^{11}$

The second class of stars, which are of order solar mass, burn ${ }^{3}$ He in steady-state. These stars go through the deuterium burning phase rapidly, converting essentially all deuterium into ${ }^{3} \mathrm{He}$. These star will then contract and heat up, eventually igniting ${ }^{3} \mathrm{He}$ through the fusion reaction Eq. (19). This reaction provides over half of the energy release from the Sun, and yet it is far slower than deuterium fusion due to the larger Coulomb barrier. We expect that ${ }^{3} \mathrm{He}$ burning to not begin in earnest until the star has collapsed nearly into its steady-state size and temperature, leading to a main sequence star that will burn for billions of years.

\section{Supernovae and Populating the ISM}

In our Universe, supernovae are vital to provide a mechanism to disperse heavy elements created inside a star out into the interstellar medium (ISM) that can later gravitationally contract to form second-generation stars and planets. The final stages of stellar evolution are therefore important to understand in the Weakless Universe in order to determine exactly what happens to dying stars and consequently to what extent the interstellar medium can be populated with elements heavier than those created during BBN.

In our Universe, stars slightly heavier than our Sun end in supernovae whose explosions release heavy elements into the interstellar medium. There are two main classes of supernovae: corecollapse (type Ib, Ic, II) and accretion (type Ia). In very massive stars, the core increasingly becomes unfusable iron supported by electron degeneracy pressure. Once the core temperature of a very massive star gets high enough, electron degeneracy pressure fails due to the electrons becoming relativistic. This is the Chandrasekhar bound for the core of the star, $1.4 M_{\odot}$. Electron degeneracy pressure fails at $T \simeq m_{e} \sim 0.5 \mathrm{MeV}$, which is coincidentally nearly the same temperature needed to have electrons recombine with protons to form neutrons, i.e., inverse $\beta$-decay (also known as the Urca process in astrophysics).

In the Weakless Universe, the electron degeneracy pressure of the core does indeed fail for temperatures beyond $T \sim m_{e}$, but obviously inverse $\beta$-decay is absent. This means that stellar

\footnotetext{
${ }^{10}$ We are grateful to Adam Burrows for performing this simulation.

${ }^{11}$ For simplicity, this simulation was performed at zero metallicity, but increasing the metallicity is only expected to modestly lower the mass of stars that burn deuterium for a long time.
} 
cores of very massive stars will collapse and, for a mass range similar to our Universe, the collapse will halt when the density reaches nuclear density. The collapse releases a huge amount of energy, and the abrupt halt at nuclear density leads to a shock wave, just as in our Universe. Unlike our Universe, however, the core has no mechanism to cool since there is no neutrino emission. This leaves the core at high temperature and pressure that can support the outer layers of the star, leading to core-collapse supernova that "fizzle". ${ }^{12}$ Notice also that final stage of these stars leads either to a black hole or a stable nuclear-density object that contains roughly equal numbers of protons and neutrons at nuclear density. This is a rather unusual astrophysical object! ${ }^{13}$

Accretion supernovae, however, lead to explosions in the Weakless Universe. Here one imagines a star with a mass that is slightly below the Chandrasekhar limit, but acquires mass through accretion with, say, a binary companion. These white-dwarf stars have burned up to about carbon and oxygen, and are marginally stable due to electron degeneracy pressure. Once accretion pushes the mass of these stars beyond the Chandrasekhar limit, gravity wins over electron degeneracy, and these stars collapse in a huge fusion explosion with no significant fraction of energy released into neutrinos. Again, the details of the explosion are quite different in the Weakless Universe versus our Universe because of the absence of the Urca process and its effects on rates of nuclear chains that occur during the explosion.

The Weakless Universe therefore appears to have one explosive supernova mechanism for populating the ISM with elements well above helium. There are also less violent processes, such as novae, which lead to large ejections of the outer layers of stars thought to arise from binary systems in which the two stars orbit each other very closely. Each nova can release as much as $0.1 \%$ of the mass of a star, and so while the mass release pales in comparison to a supernova, the far greater number of novae allow this process to potentially populate the ISM with at least some of the products of stellar nucleosynthesis.

\section{A Natural Value of the Cosmological Constant?}

We have shown that even with electroweak breaking at the Planck scale, a habitable universe can result so long as we are able to adjust technically natural parameters. It would be interesting to perform the same procedure for the cosmological constant (CC). Here our goal is far more modest than in our previous discussion: we simply wish to examine whether large scale structure and complex macroscopic systems can result if the cosmological constant is pushed to the Planck scale while we freely adjust other parameters. Performing a thorough analysis of this question is beyond the scope of this work. We will instead simply sketch some of the issues by examining simplified toy models. We find an upper bound on the CC from two qualitative requirements: that density perturbations grow, and that complex macroscopic systems consist of a large number of particles.

In order for structure to be formed in our Universe, a period of matter domination is vital to allow for linear growth of density perturbations. Matter domination is cutoff by CC domination, which is just the Weinberg bound on the anthropic size of the cosmological constant. Naively we

\footnotetext{
${ }^{12}$ If axions were present in the Weakless Universe with a sufficiently large coupling, they could lead to cooling of the supernova core that could be tuned to be equivalent to the cooling resulting from neutrinos.

${ }^{13}$ We thank D. Anderson and M. Sher for discussions on this point.
} 
could raise $\delta \rho / \rho$ up to order one, so that the bound on the cosmological constant relaxes to

$$
\rho_{\Lambda} \lesssim T_{\mathrm{eq}}^{4}
$$

But even this modest gain (about 10 orders of magnitude of 120) is much too optimistic. For Universes qualitatively similar to ours, Refs. $[4,6]$ found other astrophysical constraints limit the size of density perturbations (and place constraints on other parameters, such as the baryon density) such that the largest relaxation of the $\mathrm{CC}$ is closer to about 3 orders of magnitude of 120 . Hence, even varying multiple cosmological parameters simultaneously, this appears to be as far as one can go without radically changing the Standard Model itself.

Suppose we generalize the Standard Model to an arbitrary effective theory with matter and radiation. Could the bound on the cosmological constant be further relaxed by adjusting the temperature of matter-radiation equality in this generalized theory? Consider a toy model in which the matter is characterized by a single scale $\mu$ that sets the mass of the particles, given by

$$
\mu \equiv \epsilon M_{\mathrm{Pl}}
$$

where $\epsilon$ is sufficiently small so that we can analyze this theory perturbatively. The weakest bound on the cosmological constant arises when the matter asymmetry (analogous to $\eta_{b}$ in our Universe) is order one, so that $T_{\text {eq }} \sim \mu$. Hence, the bound given by Eq. (37) can be easily avoided by increasing $\mu$, causing matter domination to occur at an earlier epoch in this universe.

However, this is not the only bound on the vacuum energy. In particular, density perturbations on scales larger than the horizon do not grow. The largest scale that will collapse in any universe is the scale that enters the horizon as the universe is becoming CC dominated. Therefore, there is a maximal mass for any structure [20] that is given by Eq. (15), which we rewrite here as

$$
M^{\max } \sim M_{\mathrm{Pl}}^{3} \rho_{\Lambda}^{-1 / 2}
$$

for $\delta_{0}=1$. Given any field theory, there will be some minimal mass, $M_{\star}$, for interesting structure to form. For example, in our Universe, a star which is below a few percent of a solar mass does not ignite. ${ }^{14}$ A universe in which all of the structure is less than this critical mass is probably anthropically uninteresting. We can express the the critical mass $M_{\star}$ as

$$
M_{\star} \equiv N \mu,
$$

where $N$ itself is a complicated function of $\mu$. Requiring that $M_{\star} \lesssim M^{\max }$ we obtain the following bound on the $\mathrm{CC}$,

$$
\rho_{\Lambda} \lesssim \frac{M_{\mathrm{Pl}}^{6}}{M_{\star}^{2}}=\frac{M_{\mathrm{Pl}}^{6}}{N^{2} \mu^{2}}
$$

Regardless of its detailed dependence on the model, $N$ is the number of particles that make up a complex, macroscopically interesting dynamical system and is thus expected to be huge.

In our Universe with $\delta \rho / \rho \sim 1$, the bound Eq. (37) is still more restrictive than Eq. (41) when applied to structures such as galaxies and stars. For example, for $M_{\star}$ equal to the mass of a typical

\footnotetext{
${ }^{14}$ This is also true in the Weakless Universe.
} 
galaxy, the bound becomes roughly $10^{-100} M_{\mathrm{Pl}}^{4}=(1 \mathrm{keV})^{4}$. For $M_{\star}=M_{\odot}$, the bound on $\rho_{\Lambda}$ is $10^{-80} M_{\mathrm{Pl}}^{4}=(10 \mathrm{MeV})^{4}$. Nevertheless, this is obviously far smaller than the natural value, $M_{\mathrm{Pl}}^{4}$.

For the simple example under consideration we can combine Eqs. (37) and (41) to obtain

$$
\rho_{\Lambda} \lesssim \min \left\{\epsilon^{4}, \frac{1}{\epsilon^{2} N^{2}}\right\} \times M_{\mathrm{Pl}}^{4}
$$

We find that by assuming $N$ is very large, as expected for a macroscopic system, and $\epsilon$ is small, the upper bound for the vacuum energy is always parametrically smaller than $M_{\mathrm{Pl}}^{4}$.

Here we have assumed an extremely simple model that has just one scale, $\mu$. However, an essentially similar set of bounds was derived in [20] for our Universe that has several important scales (i.e., proton mass, dark matter, etc.). Tough we cannot exclude the possibility the models that violate this bound exist, we find it telling that the Planck scale seems out of reach. Taking the limit $\Lambda \rightarrow M_{\mathrm{Pl}}$ forces us into $\epsilon \rightarrow 1$ and thus $N \rightarrow 1$. It seems hard to imagine that any habitable universe results from structures that consist of single particles.

\section{Discussion}

In this paper we have constructed a Universe without weak interactions that undergoes BBN, matter domination, structure formation, star formation, long periods of stellar burning, stellar nucleosynthesis up to iron, star destruction by supernovae, and and dispersal of heavy elements into the interstellar medium. These properties of the Weakless Universe were shown by a detailed analysis that matched to our Universe as closely as possible by arbitrarily adjusting Standard Model and cosmological parameters. The Weakless Universe therefore provides a simple explicit counter-example to anthropic selection of a small electroweak breaking scale, so long as we are allowed to simultaneously adjust technically natural parameters relative to our observed Universe. As an aside, we are unaware of any obstruction to obtain a "partial Weakless Universe" in which $v<\tilde{v}<M_{\mathrm{Pl}}$ while allowing analogous adjustments of technically natural parameters.

This hypothetical universe is a counter-example to anthropic selection of the electroweak scale in the context of an effective field theory, where we are free to imagine arbitrary adjustments in technically natural parameters. An ultraviolet completion, however, may or may not permit these parameter adjustments, and as a result the Weakless Universe may or may not be "accessible". This requires detailed knowledge of the ensemble of universes that are predicted. String theory indeed appears to contain a huge number of vacua, a "landscape" [27, 28, 29, 30], in which some parameters adjust from one vacuum to another. Furthermore, only a specific set of parameters vary in the field theory landscapes considered in [31]. In its most celebrated form, the string landscape provides a potential anthropic rationale for the size of the cosmological constant [3]. However, reliable model-independent correlations between the size of the $\mathrm{CC}$ and other parameters is lacking, and so we have no way to know yet whether the variation of parameters discussed here is realized on the string landscape.

It is instructive to compare the qualitative result of simultaneously varying several parameters with respect to the $\mathrm{CC}$ and contrasting this with the electroweak scale. For a universe that 
is closely related to ours, Refs. [4, 6] showed that variations of other cosmological parameters, including $\delta \rho / \rho$ and $\eta_{b}$, lead to roughly the same bound on the cosmological constant as Weinberg's original argument. In other words, Weinberg's prediction that the CC should be near to the anthropic boundary is not significantly affected by variations of at least some other (cosmological) parameters. The electroweak scale, by contrast, can be "relaxed" all the way up to the Planck scale with the parameter adjustments that characterize the Weakless Universe. Even for a generalized universe characterized by matter with a single mass scale, requiring large scale structure be made up of large numbers of matter particles is enough to show that the cosmological constant must be parametrically smaller than the Planck scale. We conclude that the fine-tuning problems associated with the electroweak breaking scale and the cosmological constant appear to be qualitatively different from the perspective of obtaining a habitable universe.

\section{Acknowledgments}

We acknowledge numerous colleagues for valuable discussions, particularly D. Anderson, Z. Chacko, N. Dalal, S. Dimopoulos, S. Hsu, M. Kamionkowski, I. Klebanov, H. Murayama, M. Papucci, M. Peskin, K. Rajagopal, E. Ramirez-Ruiz, M. Schwartz, M. Sher, B. van Kolck, and J. Wacker. We especially thank A. Burrows for running his code with a high deuterium abundance. GDK and GP thank the Aspen Center for Physics, Stanford Linear Accelerator Center, and the Institute of Theoretical Physics at Technion University for hospitality where part of this work was completed. GDK is grateful to Lawrence Berkeley Laboratory for hospitality where this work was initiated. This work was supported in part by DOE under contracts DE-AC02-76SF00515 (RH), DE-FG0685ER40224 (GDK), and DE-AC02-05CH11231 (GP).

\section{References}

[1] B. J. Carr and M. J. Rees, Nature 278, 605 (1979).

[2] J.D. Barrow and F.J. Tipler, "The anthropic cosmological principle" Oxford University Press (1986).

[3] S. Weinberg, Phys. Rev. Lett. 59, 2607 (1987).

[4] M. Tegmark and M. J. Rees, Astrophys. J. 499, 526 (1998) [arXiv:astro-ph/9709058].

[5] A. Aguirre, Phys. Rev. D 64, 083508 (2001) [arXiv:astro-ph/0106143].

[6] M. Tegmark, A. Aguirre, M. Rees and F. Wilczek, Phys. Rev. D 73, 023505 (2006) [arXiv:astro$\mathrm{ph} / 0511774]$.

[7] M. L. Graesser, S. D. H. Hsu, A. Jenkins and M. B. Wise, Phys. Lett. B 600, 15 (2004) [arXiv:hep-th/0407174].

[8] B. Feldstein, L. J. Hall and T. Watari, Phys. Rev. D 72, 123506 (2005) [arXiv:hep-th/0506235].

[9] L. J. Hall, T. Watari and T. T. Yanagida, arXiv:hep-th/0601028. 
[10] V. Agrawal, S. M. Barr, J. F. Donoghue and D. Seckel, Phys. Rev. D 57, 5480 (1998) [arXiv:hep-ph/9707380].

[11] T. E. Jeltema and M. Sher, Phys. Rev. D 61, 017301 (2000) [arXiv:hep-ph/9905494].

[12] H. Oberhummer, A. Csoto and H. Schlattl, Nucl. Phys. A 689, 269 (2001) [arXiv:nuclth/0009046].

[13] C. J. Hogan, arXiv:astro-ph/0602104.

[14] J. F. Donoghue, E. Golowich and B. R. Holstein, "Dynamics of the standard model," Camb. Monogr. Part. Phys. Nucl. Phys. Cosmol. 2, 1 (1992).

[15] D. B. Kaplan and I. R. Klebanov, Nucl. Phys. B 335, 45 (1990).

[16] B. F. Gibson and E. V. Hungerford, Phys. Rept. 257 (1995) 349.

[17] A. G. Cohen and D. B. Kaplan, Nucl. Phys. B 308, 913 (1988).

[18] H. Davoudiasl, R. Kitano, G. D. Kribs, H. Murayama and P. J. Steinhardt, Phys. Rev. Lett. 93, 201301 (2004) [arXiv:hep-ph/0403019].

[19] R. V. Wagoner, Astrophys. J. 179, 343 (1973); L. Kawano, FERMILAB-PUB-92-004-A.

[20] S. Hellerman and J. Walcher, arXiv:hep-th/0508161.

[21] Information and tables on isotopes taken from LBNL Isotopes Project, http://ie.lbl.gov/systematics.html.

[22] S. P. Oh and Z. Haiman, arXiv:astro-ph/0108071.

[23] C. Hayashi, Ann. Rev. Astron. Astrophys. 4, 171 (1966).

[24] A. Burrows and J. Liebert, Rev. Mod. Phys. 65, 301 (1993); A. Burrows, W. B. Hubbard, J. I. Lunine and J. Liebert, arXiv:astro-ph/0103383; A. Burrows et al., Astrophys. J. 491, 856 (1997) [arXiv:astro-ph/9705201].

[25] G. Chabrier and I. Baraffe, arXiv:astro-ph/9704118.

[26] Adam Burrows, private comunication.

[27] R. Bousso and J. Polchinski, JHEP 0006, 006 (2000) [arXiv:hep-th/0004134].

[28] S. Kachru, R. Kallosh, A. Linde and S. P. Trivedi, Phys. Rev. D 68, 046005 (2003) [arXiv:hepth/0301240].

[29] L. Susskind, arXiv:hep-th/0302219.

[30] M. R. Douglas, JHEP 0305, 046 (2003) [arXiv:hep-th/0303194]; M. R. Douglas, arXiv:hep$\mathrm{ph} / 0401004$.

[31] N. Arkani-Hamed, S. Dimopoulos and S. Kachru, arXiv:hep-th/0501082. 